\title{
A Phototransistor-Based High-Sensitivity Biosensing System Using 650-nm Light
}

\author{
Yu-Wei Chang, Yu-Ting Tai, Yang-Tung Huang, Member, IEEE, and Yuh-Shyong Yang
}

\begin{abstract}
A miniature optical biosensing system based on a PMOS phototransistor and absorption photometry is proposed. The phototransistor was manufactured in a standard $0.35-\mu \mathrm{m}$ CMOS process, and it exhibited a responsivity higher than $1000 \mathrm{~A} / \mathrm{W}$ for $650-\mathrm{nm}$ light. For biochemical applications, the $\mathrm{TMB} / \mathrm{H}_{2} \mathrm{O}_{2} / \mathrm{HRP}$ method was adopted as a useful basis in our system. A sample volume of only $10 \mu \mathrm{l}$ was required to be dropped on the slide above the phototransistor. Experimental results demonstrated that a high sensitivity of $2.5 \mu \mathrm{A} / \mathrm{pM}$ was achieved, and the minimum HRP concentration successfully detected was $2.7 \mathrm{pM}$. This detection limit is three orders of magnitude better than that of a lately reported silicon biosensor, and is even comparable to that of a commercial spectrophotometer.
\end{abstract}

Index Terms-Biomedical transducers, medical diagnosis, photodetectors, phototransistors.

\section{INTRODUCTION}

A new generation of biochemical detection is expected worldwide owing to the rapid progress of biotechnology and microelectronics in the last decade. Among various kinds of detection methods, the detection of optical properties changed by chemical reactions is a competent approach to examine various important biological molecules [1], [2].

Horseradish peroxidase (HRP) is a popular enzyme that has been widely used in biochemical applications. While an antibody is used to recognize a target protein of interest, HRP can be conjugated to the antibody to serve as a label for determining the amount of the target [3]. HRP is usually used combining with hydrogen peroxide $\left(\mathrm{H}_{2} \mathrm{O}_{2}\right)$ to oxidize an added substrate that is luminescent or chromogenic. Reported optical methods for HRP detection include the luminol $/ \mathrm{H}_{2} \mathrm{O}_{2} / \mathrm{HRP}$ reaction [4], the $\mathrm{ABTS} / \mathrm{H}_{2} \mathrm{O}_{2} / \mathrm{HRP}$ reaction [5], and the $\mathrm{TMB} / \mathrm{H}_{2} \mathrm{O}_{2} / \mathrm{HRP}$ reaction [6]. The end products of these reactions can either emit or absorb the light of specific wavelengths for analysis.

Manuscript received July 31, 2008; revised October 05, 2008; accepted November 11, 2008. Current version published May 01, 2009. The associate editor coordinating the review of this paper and approving it for publication was Prof. Evgeny Katz.

Y.-W. Chang is with the Department of Electronics Engineering, Institute of Electronics, National Chiao Tung University, Hsinchu, Taiwan (e-mail: jameschang.ee93g@nctu.edu.tw).

Y.-T. Tai and Y.-S. Yang are with the Department of Biological Science and Technology, National Chiao Tung University, Hsinchu, Taiwan (e-mail: donnasafin@ hotmail.com; ysyang@ mail.nctu.edu.tw).

Y.-T. Huang is with the Department of Electronics Engineering, Institute of Electronics, and the Department of Biological Science and Technology, National Chiao Tung University, Hsinchu, Taiwan (e-mail: huangyt@ @cc.nctu.edu. tw).

Color versions of one or more of the figures in this paper are available online at http://ieeexplore.ieee.org.

Digital Object Identifier 10.1109/JSEN.2009.2020675
For commercial instruments, a photomultiplier tube usually serves as the optical sensor in a spectrophotometer. Despite its high sensitivity, the spectrophotometer has limited applications in home care instruments because of its bulky size, high cost, and high voltage (about 1000 V) [7]. Since the development of a portable, reliable, inexpensive, and convenient biosensor is the most important niche in the health care industry [8], the siliconbased biosensing system with advantages of low cost and high throughput has become an attractive candidate for personalized diagnostic kits.

A conventional CMOS photodiode can easily be formed by utilizing a single $\mathrm{N}^{+} / \mathrm{P}_{\text {well }}, \mathrm{P}^{+} / \mathrm{N}_{\text {well }}$, or $\mathrm{N}_{\text {well }} / \mathrm{P}_{\text {sub }}$ junction, but the responsivity is low [9]. Avalanche multiplication is a way to bring an internal current gain. However, the demand for high bias voltage also limits its use in many applications. Circumventing this difficulty, a CMOS phototransistor could have a current amplification under moderate bias through the internal transistor action [10].

In this research, a high-sensitivity phototransistor manufactured in a standard CMOS technology is proposed. Based on this phototransistor, a miniature optical biosensing system is developed. The optoelectronic measurements and biochemical experiments are also presented.

\section{PRINCIPLES AND METHODS}

\section{A. Biochemical Reaction}

The TMB $/ \mathrm{H}_{2} \mathrm{O}_{2} / \mathrm{HRP}$ reaction is adopted as the basis for biomedical applications in our system. TMB stands for $3,3^{\prime}, 5,5^{\prime}$-tetra-methyl-benzidine. It is neither mutagenic nor carcinogenic [11] and is a widely used reagent for ELISA (enzyme-linked immuno-sorbent assay).

The oxidation of TMB by $\mathrm{H}_{2} \mathrm{O}_{2}$ with HRP can be easily observed for qualitative analysis. The reactant solution is visually light green, whereas the soluble end product is deep blue. After the addition of sulfuric acid $\left(\mathrm{H}_{2} \mathrm{SO}_{4}\right)$ to the media, this reaction would be stopped and result in a yellow product [12]. The process can be expressed as [13]

$$
\mathrm{TMB} \stackrel{\mathrm{H}_{2} \mathrm{O}_{2}+\mathrm{HRP}}{\longrightarrow}>\mathrm{TMB}^{+} \stackrel{\mathrm{H}_{2} \mathrm{SO}_{4}}{\longrightarrow}>\mathrm{TMB}^{2+} \text {. }
$$

\section{B. Absorption Photometry}

For quantitative analysis, the analyte concentration can be evaluated by detecting the optical absorbance of the solution. The absorption spectra obtained from a standard spectrophotometer (Hitachi U-3310) show that the reactant TMB has an absorption peak at $280 \mathrm{~nm}$, whereas the blue product $\mathrm{TMB}^{+}$ 

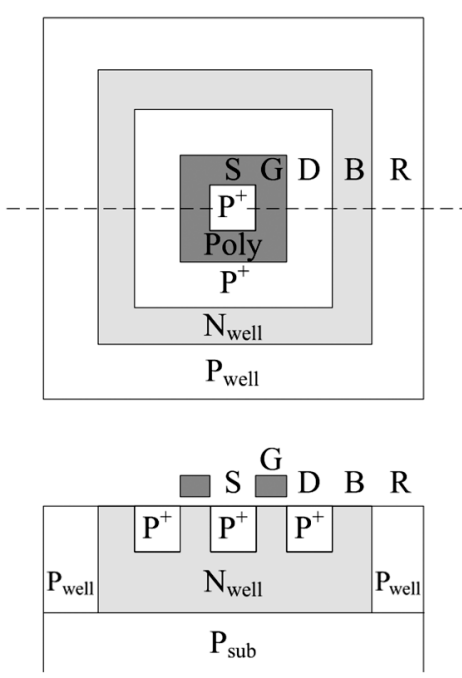

Fig. 1. The top view and cross section of the PMOS phototransistor.

has absorption peaks at 370 and $650 \mathrm{~nm}$. With the presence of $\mathrm{H}_{2} \mathrm{SO}_{4}$, the yielded yellow product $\mathrm{TMB}^{2+}$ has an absorption peak at $450 \mathrm{~nm}$.

The absorbance $A$ of the materials is described by the Beer-Lambert law [2]

$$
A=-\log \left(\frac{I}{I_{0}}\right)=\epsilon_{\lambda} \cdot l \cdot c \Longrightarrow A \propto c
$$

where $I_{0}$ and $I$, respectively, denote the initial light intensity and the light intensity after passing through the material, $\epsilon_{\lambda}$ is the wavelength-dependent molar absorptivity in units of $1 \cdot \mathrm{mol}^{-1}$. $\mathrm{cm}^{-1}, l$ is the path length in units of $\mathrm{cm}$, and $c$ is the concentration of absorbing species in the material in units of $\mathrm{M}$ $\left(1 \mathrm{M}=1 \mathrm{~mol} \cdot \mathrm{l}^{-1}\right)$. When the light source and the path length are fixed, the absorbance would be proportional to the analyte concentration. Therefore, detecting the optical signals modulated by the biochemical reactions is an efficient way to quantitate the analyte of interest.

Regarding a commercial spectrophotometer, the used sample volume and the path length are about $1 \mathrm{ml}$ and $1 \mathrm{~cm}$, respectively. Referring to (2), when a shorter path length is used, the change in the absorbance becomes smaller, which makes the detection of concentration more difficult. For a miniature system, in order to further reduce the sample volume as well as the path length, the development of a high-sensitivity optical sensor is of great importance.

\section{PMOS Phototransistor}

The top view and cross section of the proposed PMOS phototransistor are shown in Fig. 1. The $\mathrm{P}^{+}$source placed in the center is surrounded by the polysilicon gate and the $\mathrm{P}^{+}$drain, while the $\mathrm{N}_{\text {well }}$ bulk (B) is enclosed by the $\mathrm{P}_{\text {well }} \operatorname{ring}(\mathrm{R})$. It can be regarded as a PMOS with a photodiode connected across the bulk (B) and the square ring (R).

The PMOS phototransistor leaves the $\mathrm{N}_{\text {well }}$ bulk floating and uses the $\mathrm{N}_{\text {well }} / \mathrm{P}_{\text {sub }}$ junction diode for the optical access [14]. The photogenerated carriers swept into the $\mathrm{N}_{\text {well }}$ would change the bulk potential, and thereby modulate the threshold voltagem as well as the output drain current.

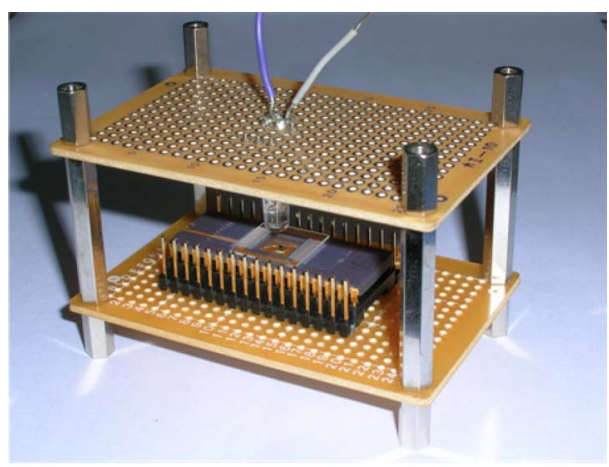

(a)

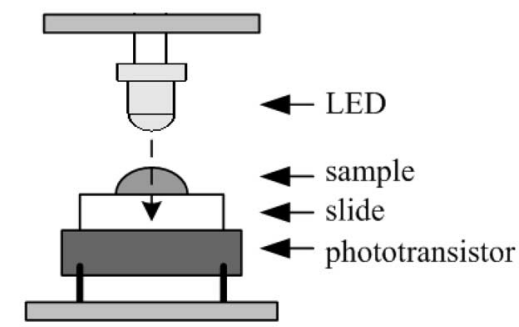

(b)

Fig. 2. The phototransistor-based biosensing system: (a) photogragh and (b) illustrated diagram.

The output drain current $I_{D}$ of a MOSFET operated in the saturation region can be expressed as

$$
I_{D}=\frac{1}{2} \frac{W}{L} \mu C_{\mathrm{ox}}\left(V_{G S}-V_{T}\right)^{2}\left(1+\frac{V_{D S}}{V_{A}}\right)
$$

where $W, L, \mu, C_{\mathrm{ox}}, V_{G S}, V_{T}, V_{D S}$, and $V_{A}$ are the channel width, the channel length, the carrier mobility, the oxide capacitance per unit area, the gate-source voltage, the threshold voltage, the drain-source voltage, and the early voltage, respectively [10]. The shift amount of the threshold voltage due to the bulk charge effect is given by

$$
\Delta V_{T}=\frac{\sqrt{2 \varepsilon_{s} q N_{B}}}{C_{\mathrm{ox}}}\left(\sqrt{2 \psi_{B}+V_{S B}}-\sqrt{2 \psi_{B}}\right)
$$

where $\varepsilon_{s}, q, N_{B}$, and $\psi_{B}$ are the permittivity of silicon, the unit electric charge, the doping concentration of the bulk, and the bulk Fermi level from the intrinsic Fermi level, respectively; $V_{S B}$ is the source-bulk voltage resulting from the optical access.

Using the proposed layout style, the sensing area of the outer ring photodiode is enlarged, so as to cause a larger shift amount of the threshold voltage. The channel length is also smaller than that of a conventional PMOS with the same area. Since the output drain current is inversely proportional to the channel length, a larger photocurrent response could be expected.

\section{Biosensing System Setup}

The proposed PMOS phototransistor with area of $100 \mu \mathrm{m} \times$ $100 \mu \mathrm{m}$ was manufactured using the TSMC (Taiwan Semiconductor Manufacturing Company) 0.35- $\mu \mathrm{m}$ standard CMOS technology. The photograph and the illustrated diagram of the whole biosensing system are shown in Fig. 2, and its dimensions are about $7 \mathrm{~cm}$ (length) $\times 5 \mathrm{~cm}$ (width) $\times 5 \mathrm{~cm}$ (height) A red LED (Centenary 31134) is used as the light source, and 


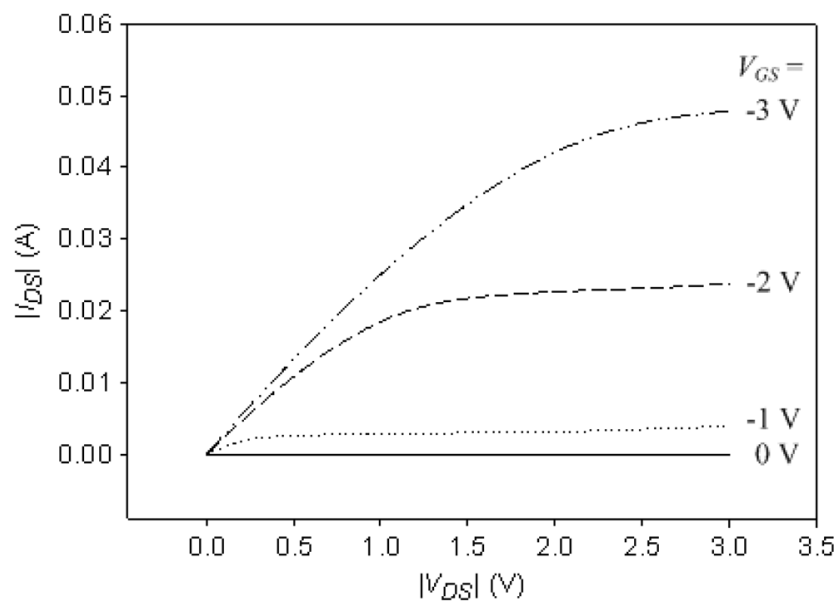

Fig. 3. The measured $I-V$ characteristics of the PMOS phototransistor.

a slide is placed above the PMOS phototransistor to hold the biochemical sample. A sample volume of only $10 \mu \mathrm{l}$ is required for the detection. The path length $l$ through the sample is about $0.1 \mathrm{~cm}$.

The light traveling through the sample is modulated and then detected by the PMOS phototransistor, which converts the modulated optical signals into current signals. The current signals are measured using a precision semiconductor parameter analyzer (HP 4156A), which is connected to a personal computer via a general purpose interface bus (GPIB). An application software (Interactive Characterization Software, ICS) is used for data acquisition.

\section{E. Reagent Preparation}

TMB liquid substrate system, HRP-streptavidin, and phosphate buffered saline (PBS) powders were purchased from Sigma. Other chemical reagents were of analytical grade and used without further purification.

The HRP stock solutions $(1 \mu \mathrm{g} / \mu \mathrm{l})$ were prepared in a PBS buffer and stored at $-20^{\circ} \mathrm{C}$. The stock enzyme solutions were melted in an ice bath just before use and diluted with double distilled water $\left(\mathrm{ddH}_{2} \mathrm{O}\right)$. The reactions were performed at $25^{\circ} \mathrm{C}$.

\section{EXPERIMENTAL RESULTS}

\section{A. Device Characteristics}

While the proposed PMOS phototransistor was without illumination, the measured $I-V$ characteristics are shown in Fig. 3. The bias conditions were as follows: the source was grounded, the drain voltage swept from 0 to $-3 \mathrm{~V}$, the gate voltage stepped from 0 to $-3 \mathrm{~V}$, the bulk was floating, and the ring voltage was fixed at $-3 \mathrm{~V}$. It can be seen that for $V_{D S}=-3 \mathrm{~V}$, the phototransistor was operated in the saturation region. The larger $\left|V_{G S}\right|$ was, the larger $\left|I_{D S}\right|$ was.

While the phototransistor was illuminated, the output drain current was modulated. For $V_{D S}=-3 \mathrm{~V}$, the measured photocurrent responses under various illumination intensities of 650-nm light are shown in Fig. 4(a). The photocurrent response divided by illumination intensity can give the responsivity, and the results are shown in Fig. 4(b). For $V_{G S}=-2 \mathrm{~V}$ and $-3 \mathrm{~V}$, the phototransistor exhibited a responsivity higher than 1000

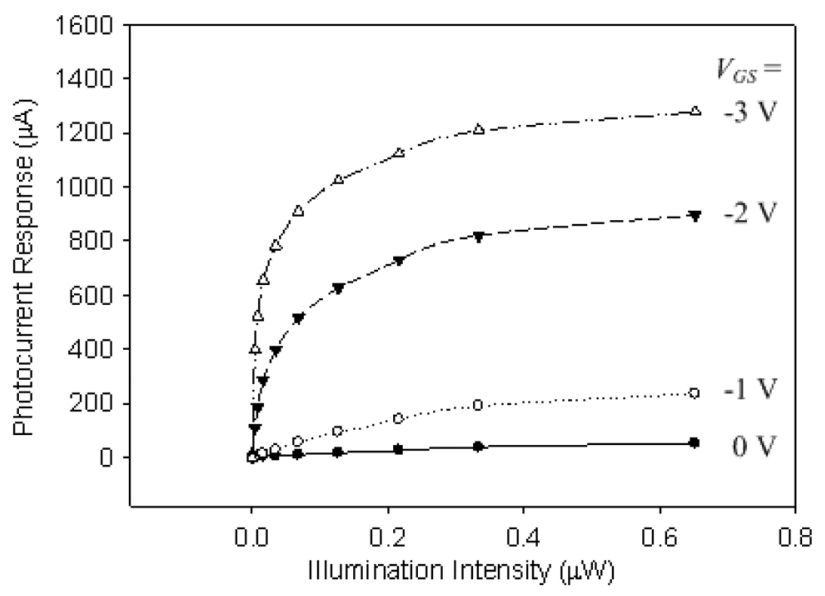

(a)

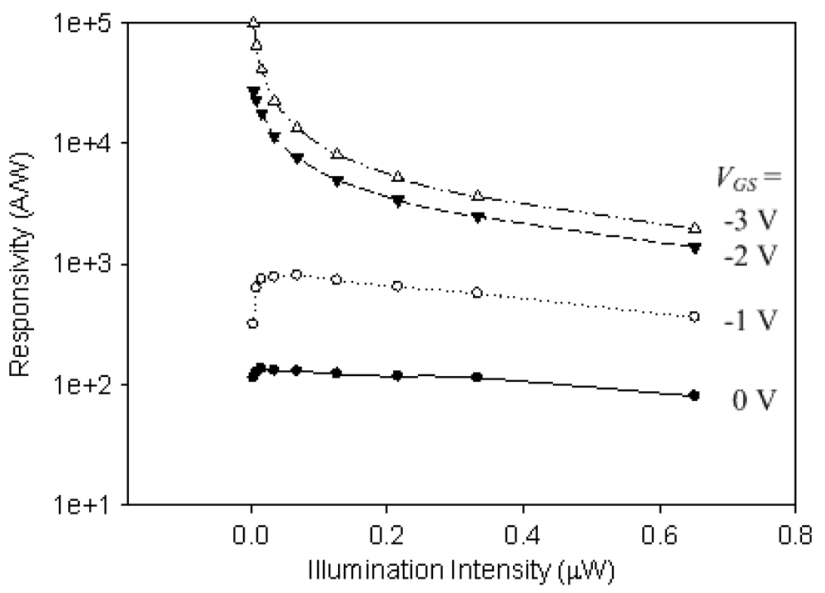

(b)

Fig. 4. The optoelectronic characteristics of the PMOS phototransistor for $V_{D S}=-3 \mathrm{~V}$ with various $V_{G S}$ : (a) photocurrent response versus illumination intensities and (b) responsivity versus illumination intensities.

A/W. The responsivity is 2000 folds higher than that of a traditional silicon $\mathrm{P} / \mathrm{N}$ junction photodiode (about $0.5 \mathrm{~A} / \mathrm{W}$ ) [7].

Even for very small bias voltages such as $V_{D S}=-0.1 \mathrm{~V}$ and $V_{G S}=0 \mathrm{~V}$, the phototransistor can successfully detected the change of illumination intensity and exhibited a responsivity of about $50 \mathrm{~A} / \mathrm{W}$.

Two measurements with an interval of four months were performed, and the device dark current were observed to examine the device stability. For $V_{D S}=-3 \mathrm{~V}$ and $V_{G S}=-2 \mathrm{~V}$, the drift amount of dark current after four months was $29.2 \mu \mathrm{A}$, which is much smaller than the amplitude of photocurrent response (at least hundreds $\mu \mathrm{A}$ ). For $V_{D S}=-0.1 \mathrm{~V}$ and $V_{G S}=$ $0 \mathrm{~V}$, the drift amount of dark current after four months was $0.75 \mathrm{nA}$, which is also much smaller than the amplitude of photocurrent response (at least hundreds nA). Hence the stability of the proposed PMOS phototransistor could be acceptable.

\section{B. Biochemical Detection}

The system setup is illustrated in Fig. 2. A sample volume of only $10 \mu \mathrm{l}$ was required to be dropped on the slide above the phototransistor. For the experiments performed using 650-nm light with different HRP concentrations, the measured photocurrent responses for $V_{D S}=-3 \mathrm{~V}$ with various $V_{G S}$ are shown in 


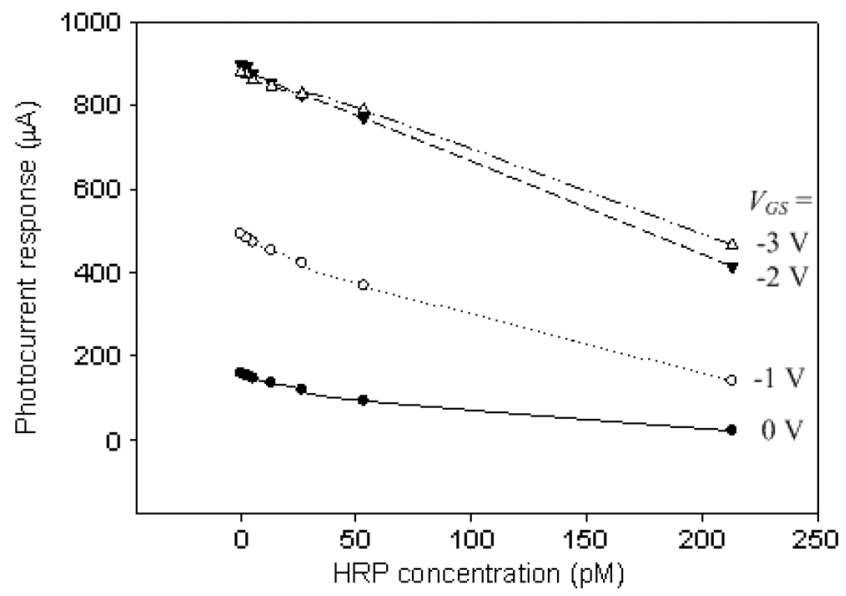

Fig. 5. The photocurrent response versus HRP concentration for $V_{D S}=-3 \mathrm{~V}$ with various $V_{G S}$.

TABLE I

THE StATISTICAL INFORMATION OF THE PHOTOCURRENT RESPONSE $I_{\mathrm{ph}}$ AND THE ABSORBANCE $A$ FOR $V_{D S}=-3 \mathrm{~V}$ AND $V_{G S}=-2 \mathrm{~V}$ UNDER VARIOUS HRP CONCENTRATION

\begin{tabular}{c|cc|cc}
\hline \multirow{2}{*}{ Conc. (pM) } & \multicolumn{2}{|c|}{$I_{\mathrm{ph}}(\mu \mathrm{A})$} & \multicolumn{2}{c}{ Absorbance } \\
\cline { 2 - 5 } & mean & SD & mean & SD \\
\hline buffer & 901.0 & 1.140 & -0.004 & 0.002 \\
2.7 & 893.2 & 2.510 & 0.017 & 0.005 \\
5.3 & 879.2 & 1.140 & 0.054 & 0.002 \\
13.3 & 857.4 & 1.643 & 0.113 & 0.004 \\
26.6 & 826.8 & 1.924 & 0.195 & 0.004 \\
53.2 & 770.8 & 1.304 & 0.346 & 0.003 \\
218.8 & 414.8 & 3.194 & 1.302 & 0.007 \\
darkroom & 0.0 & 1.342 & 2.417 & 0.003 \\
\hline
\end{tabular}

(Conc.: concentration, SD: standard deviation)

Fig. 5. The used HRP concentration values were 0.0, 2.7, 5.3, $13.3,26.6,53.2$, and $212.8 \mathrm{pM}$, respectively. Each data point is the average of five measurement results.

For $V_{D S}=-3 \mathrm{~V}$ and $V_{G S}=-2 \mathrm{~V}$, the highest sensitivity of $2.5 \mu \mathrm{A} / \mathrm{pM}$ was achieved, and the linear relationship ranged from 2.7 to $212.8 \mathrm{pM}$ with the coefficient of determination $R^{2}=0.9990$ calculated by an application software (Microsoft Excel). The statistical information of the photocurrent response $I_{\mathrm{ph}}$ is summarized in Table I. For the buffer solution, the $I_{\mathrm{ph}}$ mean is $901.0 \mu \mathrm{A}$ and the standard deviation is $1.140 \mu \mathrm{A}$. For the 2.7-pM solution, the $I_{\mathrm{ph}}$ mean is $893.2 \mu \mathrm{A}$ and the standard deviation is $2.510 \mu \mathrm{A}$. Since the difference in the $I_{\mathrm{ph}}$ mean is much larger than the standard deviation, the experimental results indicated that the minimum HRP concentration successfully detected was $2.7 \mathrm{pM}$. This detection limit is three orders of magnitude better than that of a lately reported silicon biosensor (2.4 $\mathrm{nM}$ ) [15], and is even comparable to the limit obtained from a commercial spectrophotometer (Hitachi U-3310). The required sample volume of our system is also smaller.

For $V_{G S}=0 \mathrm{~V}$, the phototransistor can sill be used for biochemical detection, and the measured $I-V$ characteristics under various HRP concentrations are shown in Fig. 6, with an arrow indicating the trend of increasing concentration. When the HRP concentration is increasing, the color of the end product become darker, which implies that more light will be absorbed, and thus the output drain current become smaller and closer to the condition in a darkroom. Even for very small bias voltages

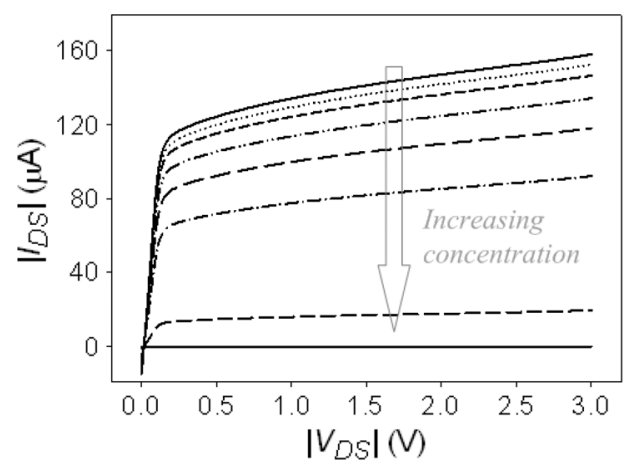

HRP Concentration

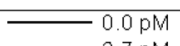

……....... $2.7 \mathrm{pM}$

----- $5.3 \mathrm{pM}$

-..-.-. $13.3 \mathrm{pM}$

$--26.6 \mathrm{pM}$

-.- - - $53.2 \mathrm{pM}$

$----212.8 \mathrm{pM}$

Darkroom

Fig. 6. The measured $I-V$ characteristics for $V_{G S}=0 \mathrm{~V}$ under various HRP concentrations.

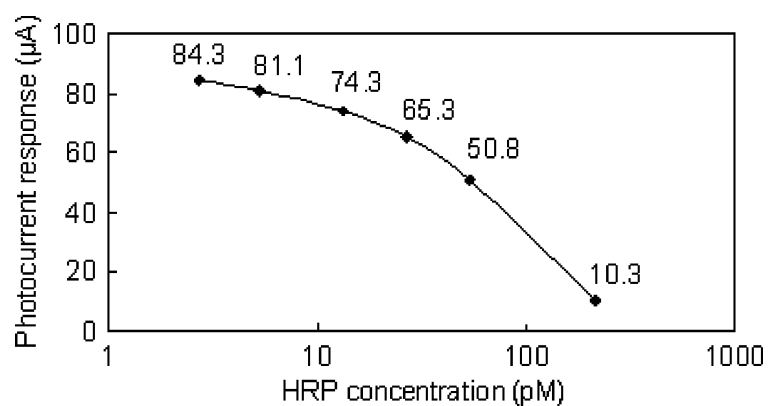

Fig. 7. The photocurrent response versus HRP concentration for $V_{D S}=$ $-0.1 \mathrm{~V}$ and $V_{G S}=0 \mathrm{~V}$.

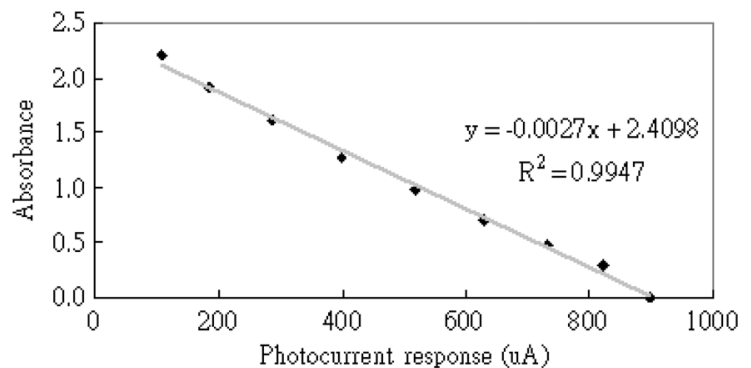

(a)

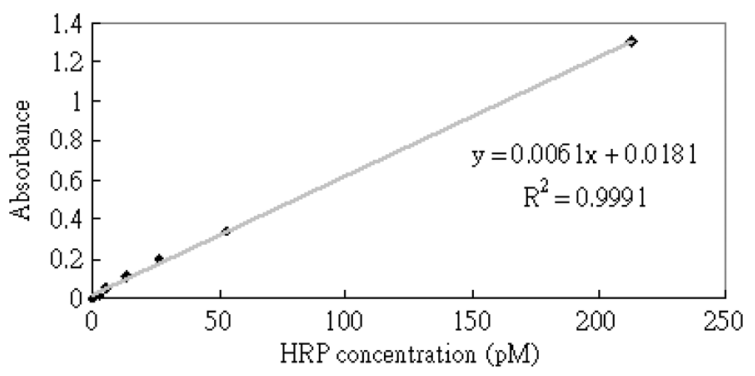

(b)

Fig. 8. The corresponding absorbance versus: (a) photocurrent response and (b) HRP concentration.

such as $V_{D S}=-0.1 \mathrm{~V}$, the experimental results shown in Fig. 7 also demonstrated a detection limit of $2.7 \mathrm{pM}$. The difference in $I_{\mathrm{ph}}$ mean between the buffer and 2.7-pM solution is $3.1 \mu \mathrm{A}$, which is much larger than the standard deviation of $0.1 \mu \mathrm{A}$.

To investigate the corresponding absorbances for various HRP concentration solutions, first, the relationship between the absorbance and the photocurrent response can be obtained by combining (2) and Fig. 4(a), and the results are shown in Fig. 8(a). 
Then, combining Figs. 5 and 8(a) gives the relationship between the absorbance and HRP concentration. For $V_{D S}=-3 \mathrm{~V}$ and $V_{G S}=-2 \mathrm{~V}$, the obtained results show that the linear detection range was from $2.7 \mathrm{pM}$ to $212.8 \mathrm{pM}$ with $R^{2}=0.9991$, which is depicted in Fig. 8(b). The statistical information of the absorbance $A$ is summarized in Table $\mathrm{I}$.

\section{CONCLUSION}

Based on absorption photometry and a PMOS phototransistor manufactured in a standard CMOS technology, a high-sensitivity biosensing system has been presented. The whole system was assembled into a compact prototype, and the TMB $/ \mathrm{H}_{2} \mathrm{O}_{2} / \mathrm{HRP}$ reaction was adopted as the basis for biomedical applications in our system. This system exhibited a high detection capability of $2.5 \mu \mathrm{A} / \mathrm{pM}$, a great detection limit of $2.7 \mathrm{pM}$, a large linear detection range from 2.7 to $212.8 \mathrm{pM}$, and a low required sample volume of $10 \mu \mathrm{l}$. Therefore, the miniature CMOS optical biosensing system has great potential toward a practical home care instrument for personalized clinical diagnosis.

\section{ACKNOWLEDGMENT}

The authors would like to thank the National Chip Implementation Center (CIC) for the assistance in chip manufacture and Ms. M.-Y. Lin for providing information on the TMB reagent.

\section{REFERENCES}

[1] R. A. Yotter, L. A. Lee, and D. M. Wilson, "Sensor technologies for monitoring metabolic activity in single cells-Part I: Optical methods," IEEE Sensors J., vol. 4, no. 4, pp. 395-411, Aug. 2004.

[2] R. Eisenthal and M. J. Danson, Enzyme Assays: A Practical Approach, 2nd ed. Oxford, U.K.: Oxford Univ. Press, 2002.

[3] N. C. Veitch, "Horseradish peroxidase: A modern view of a classic enzyme," Phytochemistry, vol. 65, pp. 249-259, 2004.

[4] W.-J. Ho, J.-S. Chen, M.-D. Ker, T.-K. Wu, C.-Y. Wu, Y.-S. Yang, Y.-K. Li, and C.-J. Yuan, "Fabrication of a miniature CMOS-based optical biosensor," Biosens. Bioelectron., vol. 22, pp. 3008-3013, 2007.

[5] Y.-W. Chang, P.-C. Yu, Y.-T. Huang, and Y.-S. Yang, "A CMOS-compatible optical biosensing system based on visible absorption spectroscopy," in Proc. IEEE Int. Conf. Electron Devices and Solid-State Circuits, 2007, vol. 2, pp. 1099-1102.

[6] P. D. Josephy, T. Eling, and R. P. Mason, "The horseradish peroxidase-catalyzed oxidation of 3,5, $3^{\prime}, 5^{\prime}$-tetramethylbenzidine," J. Biol. Chem., vol. 257, pp. 3669-3675, 1982.

[7] S. Donati, Photodetectors: Devices, Circuits, and Applications. Englewood Cliffs, NJ: Prentice-Hall, 2000.

[8] S.-H. Huang, Y.-C. Shih, C.-Y. Wu, C.-J. Yuan, Y.-S. Yang, Y.-K. $\mathrm{Li}$, and T.-K. Wu, "Detection of serum uric acid using the optical polymeric enzyme biochip system," Biosens. Bioelectron., vol. 19, pp. $1627-1633,2004$

[9] H. Zimmermamn, Integrated Silicon Optoelectronics. New York: Springer, 2000.

[10] S. M. Sze and K. K. NG, Physics of Semiconductor Devices, 3rd ed. New York: Wiley, 2007

[11] E. S. Bos, A. A. van der Doelen, N. van Rooy, and A. H. W. M. Schuurs, " $3,3^{\prime}, 5,5^{\prime}$-tetramethylbenzidine as an ames test negative chromogen for horse-radish peroxidase in enzyme-immunoassay," J. Immunoassay and Immunochemistry, vol. 2, pp. 187-204, 1981.

[12] P. Fanjul-Bolado, M. B. Gonzălez-Garcia, and A. Costa-Garcia, "Amperometric detection in TMB/HRP-based assays," Anal. Bioanal. Chem., vol. 382, pp. 297-302, 2005.

[13] B. Mecheri, L. Piras, L. Ciotti, and G. Caminati, "Electrode coating with ultrathin films containing electroactive molecules for biosensor applications," IEEE Sensors J., vol. 4, no. 2, pp. 171-179, Apr. 2004.
[14] M. Schanz, W. Brockherde, R. Hauschild, B. J. Hosticka, and A. Teuner, "CMOS photosensor arrays with on-chip signal processing," in Proc. Eur. Solid-State Circuits Conf., 1997, pp. 236-239.

[15] A. C. Pimentel, A. T. Pereira, V. Chu, D. M. F. Prazeres, and J. P. Conde, "Detection of chemiluminescence using an amorphous silicon photodiode," IEEE Sensors J., vol. 7, no. 3, pp. 415-416, Mar. 2007.

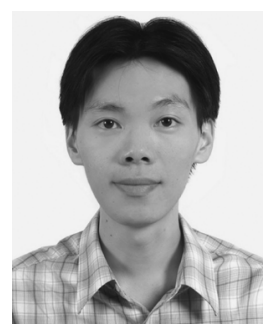

Yu-Wei Chang was born in Taichung, Taiwan, R.O.C., in 1980. He received the B.S. degree in electronics engineering from National Chiao Tung University (NCTU), Hsinchu, Taiwan, in 2002 and the M.S. degree in biophotonics engineering from National Yang Ming University, Taipei, Taiwan, in 2004. He is currently working towards the Ph.D degree at the Institute of Electronics, NCTU.

His research interests include optoelectronic devices and integrated circuits for applications in highspeed networks and biomedical diagnosis.

Mr. Change is a member of the Phi Tau Phi Scholastic Honor Society.

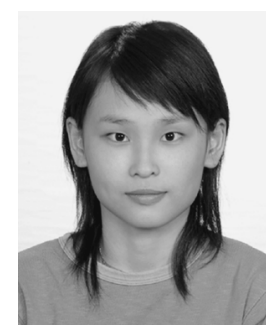

Yu-Ting Tai was born in Tainan, Taiwan, R.O.C., in 1984. She received the B.S. degree from the Department of Biological Science and Technology, National Chiao Tung University, Hsinchu, Taiwan, in 2007.

She continued her research at National Chiao Tung University as a Research Assistant. Her research interest focuses on interdisciplinary biomedical applications.

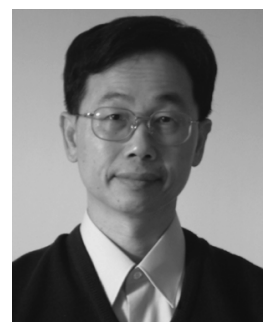

Yang-Tung Huang (M'90) was born in Taiwan, R.O.C., in 1955. He received the B.S. degree in electrophysics and the M.S. degree in electronics from National Chiao Tung University, Hsinchu, Taiwan, in 1978 and 1982, respectively, and the $\mathrm{Ph} . \mathrm{D}$. degree in electrical and computer engineering (minor in optical sciences) from the University of Arizona, Tempe, in 1990.

$\mathrm{He}$ is a Professor with the Department of Electronics Engineering and the Institute of Electronics, and a Joint Professor at the Department of Biological Science and Technology, National Chiao Tung University. He has been the Director of the Institute of Electronics for three years, the Director of Semiconductor Research Center for two years, and the Director of Nano Facility Center for four years. His current researches include integrated optics, photonic crystal waveguides, bio-optoelectronics, and optoelectronic switching networks.

Prof. Huang received the Outstanding Research Award from the National Science Council in 1998.

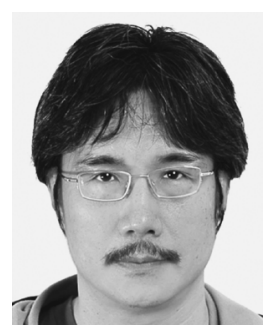

Yuh-Shyong Yang received the B.S. degree in forestry from National Taiwan University, Taipei, Taiwan, the M.S. degree in wood science and technology from the University of California, Berkeley, and the Ph.D. degree in biochemistry from the University of Wisconsin, Madison, in 1979, 1983, and 1987, respectively.

He is currently a Professor with the Department of Biological Science and Technology, National Chiao Tung University, and an Adjunct Research Fellow of the Instrument Technology Research Center and $\mathrm{Na}-$ tional Nano Device Laboratories, Taiwan. His research interests involve in the interface between biochemistry and electronics. In particular, he is interested in the specific interactions of biomolecules and their effects on electronic responses from semiconductor devices. 